\title{
Necrotizing Cutaneous Vasculitis in a Patient With Infective Endocarditis and Severe Rheumatoid Arthritis
}

\author{
Dilek Ersili ${ }^{\text {, f }}$, Ali Rlza Seren ${ }^{\text {, Emrah Koc }}{ }^{\mathrm{a}}$, Aylin Callic, Mustafa Ozmen ${ }^{\mathrm{d}}$, \\ Sezin Hlzara ${ }^{a}$ Hamza Duygu ${ }^{\mathrm{e}}$
}

\begin{abstract}
A 68-year-old woman with fever, chills, asthenia, night sweats, arthralgia, weight loss, and necrotizing skin ulcers on the body and lower extremities was admitted to our department. She had been followed for rheumatoid arthritis (RA) for over 20 years, and her rheumatoid factor (RF) level was very high at admission. A month ago, a temporary pacemaker was applied to the patient due to druginduced bradyarrhytmia, but the pacemaker's lead fractured and remained inside the right ventricle while it was removed. Revealing the vegetations on the fractured lead and tricuspid valve by echocardiography and methicillin- resistant coagulase-negative Staphylococcus aureus in separate blood cultures, the two major criteria for the diagnosis of infective endocarditis (IE) was confirmed to commence treatment of IE. The biopsy specimen from the skin ulcers revealed cutaneous vasculitis. Vasculitis is a serious complication of rheumatoid arthritis (RA) that develops in a minority of patients with this disease, and it is not uncommon in patients with IE. The diagnosis of rheumatoid vasculitis was confirmed by the history, clinical and laboratory findings of our patient. Methylprednisolon $500 \mathrm{mg}$ intravenous bolus followed by $40 \mathrm{mg}$ PO daily was administered. In spite of all taken measures the patient died of severe sepsis on the 50th day of her admission. In this case the dif-
\end{abstract}

Manuscript accepted for publication February 23, 2012

${ }^{a}$ Department of Internal Medicine, University of Katip Celebi, Faculty of Medicine Ataturk Research and Training Hospital, Izmir, Turkey

${ }^{b}$ Department of Anesthesiology and Reanimation, University of Katip Celebi, Faculty of Medicine Ataturk Research and Training Hospital, Izmir, Turkey

${ }^{c}$ Department of Pathology, University of Katip Celebi, Faculty of Medicine Ataturk Research and Training Hospital, Izmir, Turkey ${ }^{\mathrm{d}}$ Department of Rheumatology, University of Katip Celebi, Faculty of Medicine Ataturk, Research and Training Hospital, Izmir, Turkey ${ }^{\mathrm{e}}$ Department of Cardiology, University of Katip Celebi, Faculty of Medicine Ataturk, Research and Training Hospital, Izmir, Turkey ${ }^{\mathrm{f}}$ Corresponding author: Dilek Ersil, University of Katip Celebi, Faculty of Medicine and Ataturk, Research and Training Hospital, Basln Sitesi, Izmir, Turkey. Email: dileksoysal@hotmail.com

doi:10.4021/jmc591w ferential diagnosis of cutaneous vasculitis in a patient with IE and severe RA is emphasized.

Keywords: Rheumatoid artritis; Infective endocarditis; Cutaneous vasculitis

\section{Introduction}

Infective endocarditis (IE) is due to a microbial infection of the heart valves or of the endocardium in close proximity to either congenital or acquired cardiac defects [1]. In a patient with a murmur and a fever of undetermined origin, precipitating or predisposing factors, if present, should produce a high index of suspicion of infective endocarditis [2]. Infection of the pacemaker system should be suspected in any patient with an implanted device manifesting a persistent febrile illness, and if untreated, it can result in acute or subacute bacterial endocarditis and can be fatal [3]. Typical clinical features of infective endocarditis are constitutional symptoms of fever, chills, and malaise with persistent bacteremia and a new or changing heart murmur [4]. Rheumatic manifestations are known to be frequent complications of IE. Embolic complications can occur as neurologic abnormalities, arthralgias, or vasculitis and can focus attention away from the underlying cardiac cause. Such complications initially might obscure the diagnosis of endocarditis $[1,4]$.

Vasculitis is a serious complication of rheumatoid arthritis (RA) that develops in a minority of patients with this disease. The condition occurs in patients with longstanding, severe, seropositive erosive nodular RA. In one study, the mean duration between the diagnosis of RA and the onset of vasculitic symptoms was 13.6 years [5, 6]. Rheumatoid vasculitis leads to necrosis, blood vessel occlusion, and tissue ischemia in a manner that resembles other forms of systemic vasculitis, particularly polyarteritis nodosa. The classic skin lesions of rheumatoid vasculitis (RV) are deep cutaneous ulcers on the lower extremities. Clinical manifestations of small vessel vasculitis may be present in RV, but the clinical picture and most challenging problems are dominated by disease in medium-sized arteries and arterioles [5], and there is 


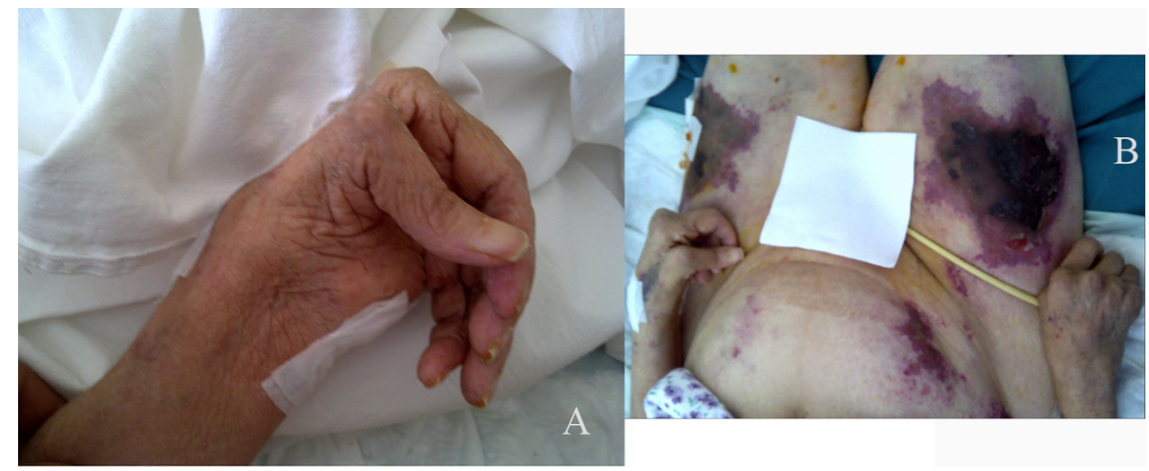

Figure 1. A: Deformation of the hands; B: Necrotising skin ulcers on the lower extremities and the body.

considerable associated morbidity and mortality [6]. In this case the differential diagnosis of cutaneous vasculitis in a patient with IE and severe RA is emphasized.

\section{Case Report}

A 68-year-old woman was admitted to our department with complaints of fever, chills, asthenia, night sweats, arthralgia, weight loss, and necrotizing skin ulcers on the body and lower extremities since the last month. She had a history of RA for 20 years and a temporary pacemaker application after a drug-induced bradyarrhytmia two months ago. The bradyarrhytmia was due to calcium channel-blocking agent (verapamil) she was using for essential hypertension. The pace-maker was removed after the drug effect was counteracted, but the pace lead fractured and remained inside the right ventricle.

At admission she was fully oriented, her axillary temperature was $38.8^{\circ} \mathrm{C}$, heart rate was $120 \mathrm{bpm}$, respiratory rate was $35 / \mathrm{min}$, and blood pressure was $135 / 95 \mathrm{mmHg}$. Physical examination revealed deformation of the hands (Fig. 1A), and feet, flexion contractures of the hip and knee, necrotizing skin ulcers on the lower extremities and the body (Fig.
1B), and borderline splenomegaly. She had normal head, eye, neck and lung findings. A 2/6 systolic murmur heard at the lower end of the sternum. Her abdomen was soft and nontender. No hepatomegaly was detected. There were no clubbing, cyanosis, and edema of the extremities.

Laboratory findings were as follows: white cell count: $46.6 \mathrm{~K} / \mu \mathrm{L}$ with neutrophils $87.1 \%$, hemoglobin: $10.4 \mathrm{~g} / \mathrm{dl}$, platelet count: $264 \mathrm{~K} / \mu \mathrm{L}$, blood urea nitrogen: $37 \mathrm{mg} / \mathrm{dl}(\mathrm{N}$ : 9.8 - 20), creatinine: $2.18 \mathrm{mg} / \mathrm{dl}(\mathrm{N}: 0.6$ - 1.1), serum albumin: $2.2 \mathrm{~g} / \mathrm{dl}(\mathrm{N}: 3.5-5)$, serum globulin: $2.2 \mathrm{~g} / \mathrm{dl}(\mathrm{N}: 1.1$ 3.1), CRP: $24.80 \mathrm{mg} / \mathrm{dl}$ (N: 0.01 - 0.82), procalcitonin: 0.215 $\mathrm{ng} / \mathrm{ml}(\mathrm{N}<0.05)$, ferritin: $719.4 \mathrm{ng} / \mathrm{ml}(\mathrm{N}: 10$ - 204), serum iron: $18 \mu \mathrm{g} / \mathrm{dl}(\mathrm{N}: 25$ - 156), serum total iron binding capacity: $110 \mu \mathrm{g} / \mathrm{dl}(\mathrm{N}: 250$ - 425), sedimentation rate: $109 \mathrm{~mm} / \mathrm{hr}$, rheumatoid factor: $929 \mathrm{IU} / \mathrm{ml}(\mathrm{N}<10)$, anti CCP: $56.5 \mathrm{U} / \mathrm{ml}$ $(\mathrm{N}<5)$.Plasma cryoglobulin and cryofibrinogen were negative. Complement levels of C3C was $86 \mathrm{mg} / \mathrm{dl}(\mathrm{N}: 83-193)$ and $\mathrm{C} 4$ was $4.3 \mathrm{mg} / \mathrm{dl}$ (N: 15 - 57). Urinalysis revealed microscopic hematuria and $2+$ proteinuria $(1.3 \mathrm{~g}$ /day by quantitative measurement). Assessment of the cell morphology showed dysmorphic red blood cells with folds and spicules. During follow-up hematuria and proteinuria resolved and no further evaluation for differential diagnosis was required.

Serology for hepatitis A, B, C, HIV, Cytomegalovirus,
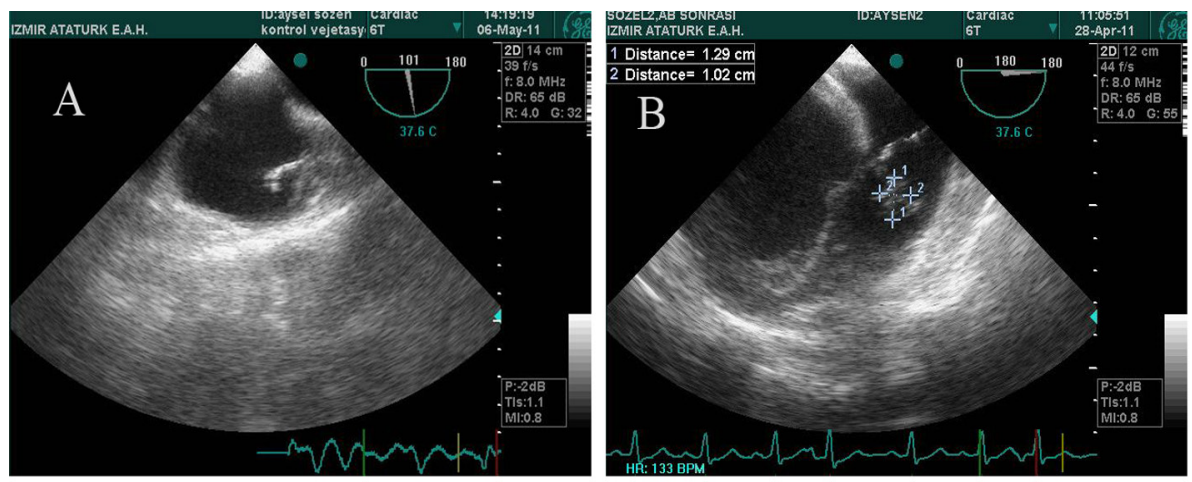

Figure 2. A: Echodensity on the fractured pacemaker lead inside the right ventricle; B: Mobile vegetation on the lateral leaflet of the tricuspid valve. 

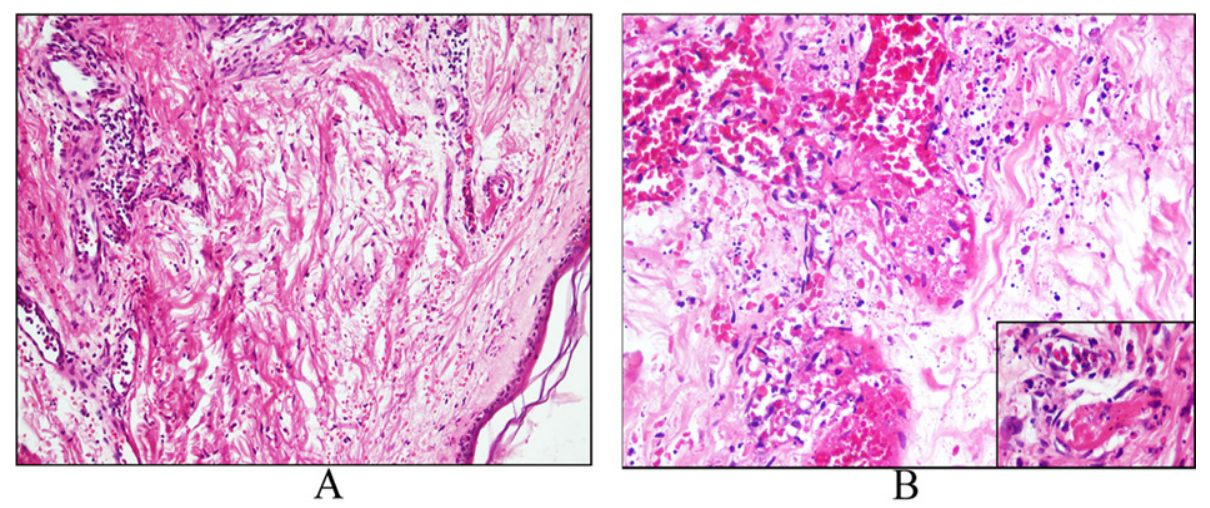

Figure 3. A: Acute vasculitis in the papillary dermis with dense inflammatory infiltrate of neutrophil-predominance; B: Fibrinoid necrosis within the vessel wall (small picture) and necrosis of the epidermis.

Epstein-Barr virus, Herpes simplex virus type 1 and 2, Toxoplasma, Rubella, Brucella, Typhoid fever, Syphilis and aspergillosis was negative.

Evaluation of antineutrophilic cytoplasmic antibodies reactive to myeloperoxidase (p-ANCA), and proteinase-3 (c-ANCA), serum antinuclear antibodies (ANA), anti-double-stranded DNA antibodies (dsDNA), other ANA and anticytoplasmic antibodies (eg, Ro (SSA), La (SSB), Smith (anti-Sm), RNP, Jo-1), antiphospholipid antibodies (eg,anticardiolipin antibodies), Scl-70 antibody (topoisomerase-1), and antibody to centromeric protein (anticentromere antibody) revealed negative.

The electrocardiogram and chest radiograph were normal. Colored doppler ultrasonography of the upper and lower extremities revealed no abnormalities in the flow and structure of the arterial and venous vascularite.

\section{Clinical course}

At admission, empiric antibiotic therapy (ceftriaxone $2 \mathrm{~g} / \mathrm{d}$ ) was commenced after blood and urine samples were obtained for cultures. In case of possible IE, blood cultures were obtained by four different vene punctures over $24 \mathrm{~h}$, at least $1 \mathrm{~h}$ apart.

On the second day of her hospital stay, transthoracic echocardiogram followed by transesophageal showed an ejection fraction of $30 \%$, an echodensity on the fractured pacemaker lead inside the right ventricule, and a mobile, $1.2 \times 1.0 \mathrm{~cm}$ vegetation on the lateral leaflet of the tricuspid valve with minimal tricuspid regurgitation (Fig. 2A, B). Antibiotherapy with vancomycin $500 \mathrm{mg}$ and imipenem $500 \mathrm{mg}$ IV daily were commenced according to the definition of possible IE by clinical criteria [4]. Antibiotic dosages were adjusted by creatinine clearance which was $27.8 \mathrm{ml} / \mathrm{min}$. Dose regimens changed as the patient's creatinine clearance improved. Methicillin- resistant coagulase-negative Staphylococcus aureus was identified in two of the four blood cul- tures drawn $>12 \mathrm{~h}$ apart, and definite IE was diagnosed by the presence of two major criteria [7, 8]. The high level of rheumatoid factor was not taken into account because of the patient's longstanding and severe RA although it is one of the minor diagnostic criteria of IE.

Response to treatment was monitored by the clinical status (in particular course of fever) and C-reactive protein. Echo follow-up examinations were carried on for later comparisons and detection of complications as proposed [7]. Creactive protein and leucocyte count fell to normal levels as the patient responded to therapy. According to the guidelines, duration of therapy is somewhat arbitrary and should be guided by the course of the disease, 4 weeks of intravenous therapy is usually considered the minimum $[7,8]$. It has been hypothesized that anticoagulatory or antiaggregatory drugs might be benefical in reducing the growth of vegetations [7]. There was no clinical evidence for such measures during follow-up. Anticoagulation may even be hazardous in view of the potential for haemorrhagic complications after cerebral embolization [7]. We did not confront with a complication of cerebral emboli. Neither an anticoagulatory nor an antiaggregatory drug was commenced during the treatment of the case. On the second week of the treatment with antibiotics, the patient's temperature raised above $38^{\circ} \mathrm{C}$ again. Candida stellatoidea was identified from the urine culture, and fluconazole $200 \mathrm{mg}$ IV daily was administered.

Histopathologic findings of the biopsy specimen from skin lesions revealed acute severe vasculitis in the papillar dermis with dense inflammatory infiltrate of neutrophilpredominance, fibrinoid necrosis within the vessel wall (Fig. 3B-small picture) and necrosis of the epidermis which was consistent with leukocytoclastic cutaneous vasculitis (LCV) (Fig. 3A, B). Differential diagnosis were considered between infection, connective tissue disease and drug reaction [5]. No drug intake was registered. In patients with septic vasculitis, the demonstrative severe pandermal LCV with neutrophiland bacteria-containing (one of the implicated microbial 
pathogens is staphyloccoccus) thrombi, extravascular neutrophilia with microabscesses and neutrophilic exocytosis, and papillary dermal edema [9] were not present in our case. The longstanding history and destructive RA with very high seropositivity achieved the diagnosis of romatoid vasculitis in our patient. Treatment administered with methylprednisolon $500 \mathrm{mg}$ IV bolus followed by $40 \mathrm{mg}$ PO daily.

It is generally accepted that large (usually defined as $>$ $10 \mathrm{~mm}$ ) mobile vegetations pose a grave embolic threat and should therefore be removed surgically as soon as possible [7]. The patient was consulted with the department of cardiovascular surgery. In our case, the patient's poor clinical condition and difficulty of moving the fractured lead away without causing recurrent emboli postponed the surgery. After four weeks of medical treatment, a repeat transesophageal echocardiogram revealed no change from the previous one. Although the patient's blood and urine cultures were negative on the 4th week of her admission, she remained on vancomycin $2 \mathrm{~g} / \mathrm{d}$ and imipenem $500 \mathrm{mg} /$ qid. Fluconazole was discontinued.

On the 5th week of her hospital stay, vancomycin resistant enteroccoccus (VRE) was identified from blood culture. She was isolated from the other patients. Vancomycin was discontinued and daptomycin (cubicin $6 \mathrm{mg} / \mathrm{kg}$ in $0.9 \%$ sodium chloride by intravenous infusion over a 30 minute period once every 24 hours for 14 days) was commenced. On the 6th week of her hospital stay, she had severe respiratory failure due to pneumonia, and Acinetobacter baumannii was identified from the sputum culture. It was sensitive to only amikacin. Right heart endocarditis in the majority of cases leads to- sometimes silent- septic pulmonary embolism and may present with signs of pneumonia and pleuritis [7]. Two days after amikacin had been commenced, the patient's clinical situation deteriorated and had to be supported by mechanical ventilation. On the 50th day of her hospital stay she died of severe sepsis.

\section{Discussion}

Infection of valvular tissue or cardiovascular endothelium by a variety of pathogens constitutes IE. Although endocarditis mostly involves the cardiac valves, it may also manifest as endarteritis or develop on foreign bodies such as intravenous lines, pacemaker leads, conduits, etc. Today the physician often confronts more aggressive infections, in particular staphylococcal endocarditis, and often the disease arises in immunocompromised patients [7].

Infective endocarditis is now affecting older patients who more often develop IE as the result of health care-associated procedures, either in patients with no previously known valve disease or in patients with prosthetic valves [8].

There is no specific laboratory marker of IE. Laboratory abnormalities include leucocytosis with granulocytosis with a left shift (or leucopenia, especially in overt sepsis), elevated sedimentation rate, elevated C-reactive protein and gamma globulin levels. Anaemia of infection with low serum iron levels is a cardinal sign of endocarditis. Circulating immune complexes and occasionally a positive rheumatoid factor are detectable. The most important laboratory test is the blood culture. IE usually leads to a continuous bacteraemia, such that (in the absence of pretreatment with antibiotics) blood cultures are very sensitive to detect the disease and may be drawn at any time independent from the time course of fever $[7,8]$.

In a pooled analysis of 3784 episodes of IE, it was shown that oral streptococci had fallen into second place to staphylococci as the leading cause of IE. Traditionally, native valve staphylococcal IE is due to S. aureus, which is most often susceptible to oxacillin, at least in community-acquired IE. In contrast, staphylococcal prosthetic valve IE is more frequently due to coagulase-negative staphylococci (CNS) with oxacillin resistance. Mortality in large series ranges between $15 \%$ and $20 \%$. Mortality is the highest in staphylococcal and fungal endocarditis [8].

Presented with the nonspecific systemic symptoms of fever of unknown origin with fever, chills, malaise, night sweats, arthralgia, and weight loss, laboratory findings with granulocytosis, anaemia of infection, high levels of C-reactive protein, ferritin, and sedimentation rate, and low levels of C4 with methicillin-resistant 1solate of Staphylococcus aureus bloodstream infection (bacteremia of which most often originates from the skin and oropharynx) and intracardiac pacemaker lead with an echodensity on it and a mobile vegetation on the tricuspid valve, the diagnosis of right-sided IE was considered in our patient. Although intravenous drug use is a well-defined risk factor for the development of rightsided IE, our patient had no previous history of intravenous drug use.

The classification of vasculitic syndromes are based on clinical, pathologic, and immunologic features [4], and diagnostic imaging studies [9]. It is well-known that patients with RA exhibit various cutaneous conditions including rheumatoid vasculitis [10]. Because proof of vasculitis is not always obtainable, even in biopsy specimens, biopsies from different lesions and from the border of the lesions were taken as recommended $[5,10]$ in our patient. The histopathologic findings were consistent with leukocytoclastic cutaneous vasculitis (LCV). As previously reported, LCV related to bacterial infection was considered if no drug intake was registered before the development of skin lesions, and bacteriologic evidence of infection was obtained [1]. In cases with IE and LCV, a trend to low serum complement levels was evident. This was associated to an immune complex-mediated process initiated by antigen products of the infectious agent responsible for the IE, or to the result of abnormal immunoregulation related to the infectious disease [1]. Although some cases of drug-induced leukocytoclastic vasculitis man- 
ifest a severe pan-dermal and/or pustular vasculitis, such as those associated with calcium channel blockers, the typical drug-induced leucocytoclastic vasculitis is mild in character and is confined to the superficial vascular plexus [9].

It is important that leg ulcers do not always mean vasculitis. Chronic leg ulcers, with or without edema are often recognized in patients with RA. The etiology is multifactorial, but often associated with venous insufficiency [10]. By using doppler ultrasonography, venous insufficiency of the lower extremities was excluded in our patient. According to Jorizzo and Daniels' classification of RV [10], our patient had severe form of RV with digital gangrene, and large cutaneous ulcers. Infection of the ulcers, if occurs will be a complicating risk. By using broad spectrum antibiotics for IE, we did not come across with any infection of the ulcers in the case.

The etiology of RV is unclear. Rheumatoid vasculitis usually develops at a time when the inflammatory arthritis is 'burned out', (ie, when the erosive process that led to joint destruction has become less active) [5]. High titers of rheumatoid factor (RF) are reported consistently to be the strongest predictor of the development of RV, and anti-cyclic citrullinated peptide (CCP) antibodies may be helpful in distinguishing RV from other forms of small vessel vasculitis. Circulating immune complexes were negative, and the RF and CCP were strongly positive in our patient. We could not look for antiendothelial cell antibodies, but, antiedothelial cell antibody assays have no defined role in making the diagnosis, assessing the prognosis, or gauging disease activity in $\mathrm{RV}$ at present [5].

Numerous medications used to treat RA have also been proposed as triggers of RV, partly because of some similarities between drug-induced hypersensitivity vasculitis and $\mathrm{RV}$. In particular, the treatment of RA with oral glucocorticoids has been implicated in the development of $\operatorname{RV}[5,6]$. Rheumatoid vasculitis most often occurs in people with at least 10 years of severe disease. Our patient had a history of RA for over 20 years, and had been on corticosteroid therapy of differrent dose regimens since then. Systemic vasculitis complicating rheumatoid arthritis appears to be on the decline. This is probably as a consequence of modern and better treatments of the underlying arthritis.

\section{Acknowledgement}

We would like to thank to the colleques of biochemistry and hormone laboratories in Ataturk Research and Training Hospital for the measurements.

\section{Conflict of Interests}

The authors declare that they have no competing interests.

\section{Grant Support}

None.

\section{Authors' Contributions}

$\mathrm{DE}$ and ARS acquired the data and wrote the manuscript, EK, MO, SH and HD performed the clinical follow-up, and $\mathrm{AC}$ performed the pathological evaluation of the patient.

\section{References}

1. Gonzalez-Juanatey C, Gonzalez-Gay MA, Llorca J, Crespo F, Garcia-Porrua C, Corredoira J, Vidan J, et al. Rheumatic manifestations of infective endocarditis in non-addicts. A 12-year study. Medicine. 2001;80(1):919.

2. Khan MG.Infective endocarditis. In: Khan MG, editor. Heart Disase Diagnosis and Therapy. 1st ed. Baltimore: Williams \& Wilkins;1996.p.461-76.

3. Saksena S, Madan N, Prakash A, Giorgberidze I. Nonpharmacologic therapy for cardiac arrhtmias: Cardiac pacing, implantable cardioverter-defibrillators, and catheter and surgical ablation. In: Khan MG, editor. Heart Disase Diagnosis and Therapy. 1st ed. Baltimore: Williams \& Wilkins;1996.p. 567-611.

4. Conti T, Barnet B. The diagnostic challenge of infective endocarditis: cutaneous vasculitis leading to the diagnosis of infective endocarditis. The Journal of the American Board of Family Practice / American Board of Family Practice. 2001;14(6):451-456.

5. Whelan P, Stone JH, Hunder GG, Romain PL. Clinical manifestations and diagnosis of rheumatoid vasculitis. UpToDate (last updated) 2011 Jan (last literature review version 2011 May). Available from:http://www.uptodate.com/contents/clinical-manifestations-and-diagnosis-of-rheumatoid-vasculitis.

6. Watts RA, Mooney J, Lane SE, Scott DG. Rheumatoid vasculitis: becoming extinct? Rheumatology. 2004;43(7):920-923.

7. Daniel WG, Flachskampf FA. Infective endocarditis. In: Camm AJ, Lüscher TF, Serruys PW, eds. The ESC textbook of Cardiovascular Medicine. 1st ed. Massachusetts: Blackwell Publishing; 2006.p.671-84.

8. Habib G, Hoen B, Tornos P, Thuny F, Prendergast B, Vilacosta I, Moreillon P, et al. Guidelines on the prevention, diagnosis, and treatment of infective endocarditis (new version 2009): the Task Force on the Prevention, Diagnosis, and Treatment of Infective Endocarditis of the European Society of Cardiology (ESC). Endorsed by the European Society of Clinical Microbiology and Infectious Diseases (ESCMID) and the International So- 
ciety of Chemotherapy (ISC) for Infection and Cancer. European heart journal. 2009;30(19):2369-2413.

9. Crowson AN, Mihm MC, Jr., Magro CM. Cutaneous vasculitis: a review. Journal of cutaneous pathology.
2003;30(3):161-173.

10. Yamamoto T. Cutaneous manifestations associated with rheumatoid arthritis. Rheumatology international. 2009;29(9):979-988. 